\title{
Pentalenic acid is a shunt metabolite in the biosynthesis of the pentalenolactone family of metabolites: hydroxylation of 1-deoxypentalenic acid mediated by CYP105D7 (SAV_7469) of Streptomyces avermitilis
}

\author{
Satoshi Takamatsu ${ }^{1,4}$, Lian-Hua $\mathrm{Xu}^{2,4}$, Shinya Fushinobu ${ }^{2}$, Hirofumi Shoun ${ }^{2}$, Mamoru Komatsu ${ }^{1}$, \\ David E Cane ${ }^{3}$ and Haruo Ikeda ${ }^{1}$
}

Pentalenic acid (1) has been isolated from many Streptomyces sp. as a co-metabolite of the sesquiterpenoid antibiotic pentalenolactone and related natural products. We have previously reported the identification of a 13.4-kb gene cluster in the genome of Streptomyces avermitilis implicated in the biosynthesis of the pentalenolactone family of metabolites consisting of 13 open reading frames. Detailed molecular genetic and biochemical studies have revealed that at least seven genes are involved in the biosynthesis of the newly discovered metabolites, neopentalenoketolactone, but no gene specifically dedicated to the formation of pentalenic acid (1) was evident in the same gene cluster. The wild-type strain of $S$. avermitilis, as well as its derivatives, mainly produce pentalenic acid (1), together with neopentalenoketolactone (9). Disruption of the sav7469 gene encoding a cytochrome P450 (CYP105D7), members of which class are associated with the hydroxylation of many structurally different compounds, abolished the production of pentalenic acid (1). The sav7469-deletion mutant derived from SUKA11 carrying pKU462:: ptl-cluster $\Delta$ ptIH accumulated 1-deoxypentalenic acid (5), but not pentalenic acid (1). Reintroduction of an extra-copy of the sav7469 gene to SUKA11 1 sav7469 carrying pKU462::ptl-cluster $\Delta$ ptIH restored the production of pentalenic acid (1). Recombinant CYP105D7 prepared from Escherichia coli catalyzed the oxidative conversion of 1-deoxypentalenic acid (5) to pentalenic acid (1) in the presence of the electron-transport partners, ferredoxin (Fdx) and Fdx reductase, both in vivo and in vitro. These results unambiguously demonstrate that CYP105D7 is responsible for the conversion of 1-deoxypentalenic acid (5) to pentalenic acid (1), a shunt product in the biosynthesis of the pentalenolactone family of metabolites.

The Journal of Antibiotics (2011) 64, 65-71; doi:10.1038/ja.2010.135; published online 17 November 2010

Keywords: biosynthesis; cytochrome P450; pentalenolactone; sesquiterpene; Streptomyces avermitilis

\section{INTRODUCTION}

Pentalenolactone is a sesquiterpenoid antibiotic first discovered in 1957 in the culture extracts of Streptomyces roseogriseus, ${ }^{1}$ and subsequently isolated from numerous Streptomyces microorganisms. ${ }^{2-4}$ Pentalenolactone is active against both Gram-positive and Gramnegative strains of bacteria, as well as pathogenic and saprophytic fungi. ${ }^{5}$ Pentalenolactone is also a potent and specific antiviral agent, inhibiting the replication of DNA viruses, such as the causal agent of herpes simplex HSV-1 and HSV-2, ${ }^{6}$ and can inhibit vascular smooth muscle cell proliferation. ${ }^{7}$ The first step in pentalenolactone biosynthesis is the cyclization of farnesyl diphosphate, the universal precursor of all sesquiterpenes, to pentalenene (2), which is the parent hydrocarbon of the pentalenolactone family of metabolites. From the results of feeding experiments for pentalenolactone biosynthesis, a variety of plausible intermediates in the conversion of pentalenene to pentalenolactone have been isolated, including 1-deoxypentalenic acid (5; as glucuronate ester), ${ }^{4}$ as well as pentalenic acid (1), ${ }^{8,9}$ a demonstrated shunt metabolite of the main pentalenolactone biosynthetic pathway.

${ }^{1}$ Laboratory of Microbial Engineering, Kitasato Institute for Life Sciences, Kitasato University, Minami-ku, Sagamihara, Kanagawa, Japan; ${ }^{2}$ Department of Biotechnology, Graduate School of Agriculture and Life Sciences, The University of Tokyo, Bunkyo-ku, Tokyo, Japan and ${ }^{3}$ Department of Chemistry, Brown University, Providence, RI, USA

${ }^{4}$ These authors contributed equally to this work.

Correspondence: Professor H Ikeda, Laboratory of Microbial Engineering, Kitasato Institute for Life Sciences, Kitasato University, 1-15-1 Kitasato, Minami-ku, Sagamihara, Kanagawa 252-0373, Japan.

E-mail: ikeda@Is.kitasato-u.ac.jp

Dedicated to the late Dr C Richard Hutchinson for his exceptional contributions to natural product biosynthesis, engineering, and drug discovery and in honor of his friendship. Received 15 August 2010; revised 16 October 2010; accepted 17 October 2010; published online 17 November 2010 


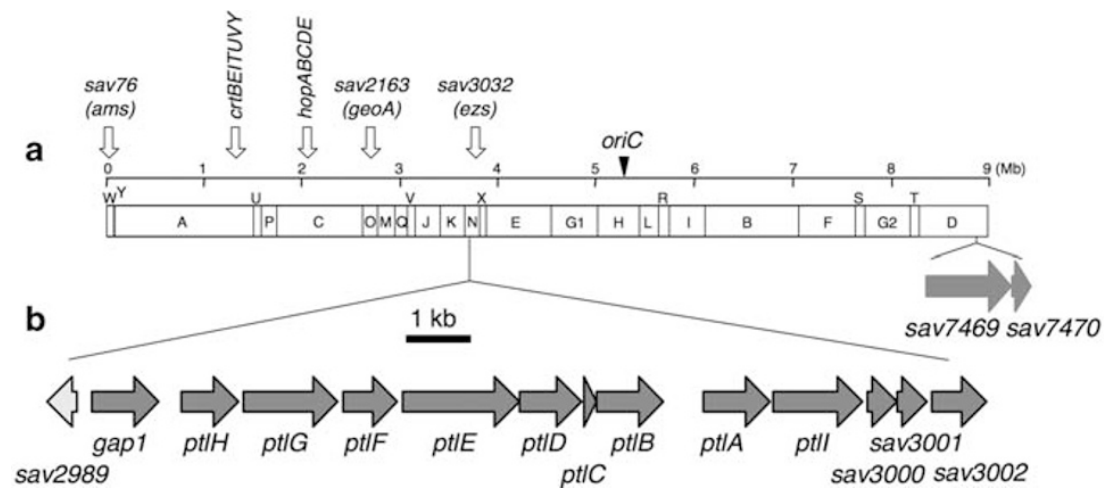

Figure 1 Asel-physical map of $S$. avermitilis and distribution of biosynthetic genes encoding terpenoid compounds (sav76, gene for avermitilol biosynthesis; crt, genes for isoneriatene biosynthesis; hop, genes for squalene and hopanoid biosynthesis; sav2163, gene for germacradienol and geosmin biosynthesis; sav3032, gene for epi-isozizaene biosynthesis) (a); and gene cluster for neopentalenoketolactone biosynthesis (sav2989, gene encoding MarR-family transcriptional regulator; gap1, gene for pentalenolactone-insensitive glyceraldehyde-3-phosphate dehydrogenase; $p t / H$, gene for 1-deoxypentalenic acid 11- $\beta$ hydroxylase; $p t / G$, gene for transmembrane efflux protein; $p t / F$, 1-deoxy-11 $\beta$-hydroxypentalenic acid dehydrogenase; ptIE, gene for Baeyer-Villiger monooxygenase; $p t I D$, gene for dioxygenase; $p t / C$, gene for hypothetical protein; $p t / B$, gene for farnesyl diphosphate synthase; ptIA, gene for pentalenene synthase; ptII, gene for pentalenene C13 hydroxylase CYP183A1; sav3000, gene for AraC-family transcriptional regulator; sav3001, gene for lyase; sav3002, gene for hypothetical protein) (b). Two genes, sav7469 and sav7470, encode CYP105D7 and FdxH, respectively.

The fact that tritium label from $(1 R)-\left[1-{ }^{3} \mathrm{H}\right]$ pentalenene $(2)$ was lost upon formation of pentalenic acid (1), but was retained in the derived pentalenolactone has definitively ruled out $\mathbf{1}$ as an intermediate in the formation of pentalenolactone. ${ }^{9}$ A plausible biosynthetic pathway for pentalenolactone biosynthesis has been proposed on the basis of the structures of these isolated pentalenolactone metabolites, but until recently, however, there had been no direct experimental evidence for biosynthetic sequence leading from pentalenene (2) to the pentalenolactone family of metabolites.

Streptomyces avermitilis is a Gram-positive soil bacterium responsible for the production of the anthelminthic macrocyclic lactone avermectins that are widely used in human and veterinary medicine. Sequencing of the complete $9.03-\mathrm{Mb}$ linear genome of $S$. avermitilis revealed at least 37 presumed biosynthetic gene clusters related to secondary metabolism, with at least six of them encoding putative terpenoid biosynthetic pathways as shown in Figure 1a. ${ }^{10-13}$ Among the latter, a $13.4-\mathrm{kb}$ gene cluster centered at $3.75 \mathrm{Mb}$ in the S. avermitilis genome and containing 13 unidirectionally transcribed open reading frames (sav2990 - sav3002) has been implicated in the biosynthesis of pentalenolactone-like metabolites (Figure 1b). Although pentalenolactone itself has not been detected in the organic extract of $S$. avermitilis, we have isolated the common pentalenolactone shunt metabolite pentalenic acid (1) from these cultures. ${ }^{14}$ Deletion of the 13.4-kb operon from $S$. avermitilis abolished the production of both neopentalenoketolactone (9) and pentalenic acid (1), whereas transfer of the entire gene cluster to Streptomyces. lividans 1326, a strain that normally does not produce pentalenolactones, resulted in generation of pentalenic acid (1). In parallel with these molecular genetic investigations, we have been systematically expressing in E. coli the individual genes from the 13.4-kb biosynthetic cluster in order to identify the natural substrates and intrinsic biochemical reactions for each open reading frame (Figure 2). Direct evidence for the function of the 13.4-kb cluster came from the expression of recombinant ptlA (sav2998), ${ }^{14}$ ptlI (sav2999), ${ }^{15}$ ptlH $\left(\right.$ sav2991) ${ }^{16,17}$ ptlF $(\operatorname{sav} 2993){ }^{18}$ and ptlE (sav2994), ${ }^{19}$ respectively. The fact that the $\Delta p t l D$ mutant of $S$. avermitilis accumulates neopentalenolactone D $(\mathbf{8})$ suggests that $\mathbf{8}$ is likely to be the natural substrate for the $p t l D$ gene product in the formation of neopentalenoketolactone $(\mathbf{9}){ }^{20}$
We have thus, elucidated all but the final step in the biosynthetic pathway for the pentalenolactone variant, neopentalenoketolactone (9), whereas the genetic and biochemical basis for the accumulation of pentalenic acid (1) in S. avermitilis remained to be clarified. The shunt product pentalenic acid (1) is thought to be formed by C-1 hydroxylation of 1-deoxypentalenic acid (5), an intermediate in the biosynthesis of the pentalenolactone and neopentalenolactone family of metabolites, presumably under the control of a suitable hydroxylase or cytochrome P450 (CYP). Significantly, however, no candidate gene encoding such a hydroxylase is evident in the $13.4-\mathrm{kb}$ ptl cluster. In fact, several S. avermitilis CYP genes are located within the biosynthetic clusters for avermectin, oligomycin, filipin, albaflavenone and neopentalenolactone biosynthesis. Other CYP genes are distributed independently throughout the linear chromosome of S. avermitilis. ${ }^{20}$ It is, therefore, of considerable interest to identify which S. avermitilis CYP gene is responsible for the conversion of 1-deoxypentalenic acid (5) to pentalenic acid (1). We now provide evidence that CYP105D7 encoded by sav7469 is responsible for hydroxylation at the C-1 position of 1-deoxypentalenic acid (5), resulting in accumulation of pentalenic acid (1) as a shunt metabolite of pentalenolactone or neopentalenolactone biosynthesis.

\section{MATERIALS AND METHODS}

\section{Bacterial strains}

The large-deletion mutants of $S$. avermitilis, SUKA5, SUKA7 (SUKA5 $\Delta$ sav7469), SUKA11 (SUKA5 $\Delta$ ptl-cluster::ermE) and SUKA13 (SUKA11 $\Delta$ sav 7469$),{ }^{21}$ were used for production of neopentalenolactone and related compounds. Culture conditions for the sporulation and regeneration of protoplasts after PEG-mediated protoplast transformation have been previously described. 22,23

\section{Construction of $\Delta p t l H$-deletion mutant}

The $\Delta p t l H$-deletion mutants were prepared using the entire $14.9-\mathrm{kb}$ S. avermitlis ptl-cluster clone (pKU462::ptl-cluster), in which ptlA had been placed under the control of the ermE promoter, as previously described, ${ }^{14,19}$ using as host strains $S$. avermitilis SUKA11 and SUKA13. In-frame $\Delta p t l H$ deletion mutant: forward primer, $5^{\prime}$-ACCACGGCCACTGAGGTCTCACAAGG AGATGAATCTGTGGCCAGTGAGTTCGAGCGACT-3' (bold characters indicate the upstream region of $p t l H$ on the chromosome and underlined characters correspond to the start codon of $p t l H$ ) and the reverse primer 


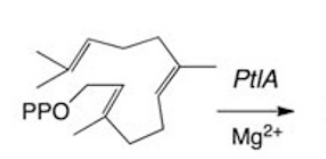

farnesyl diphosphate

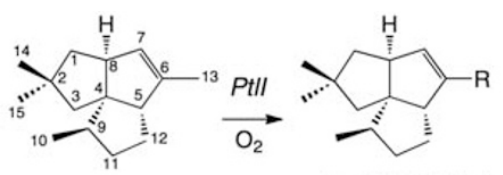

pentalenene

(2)
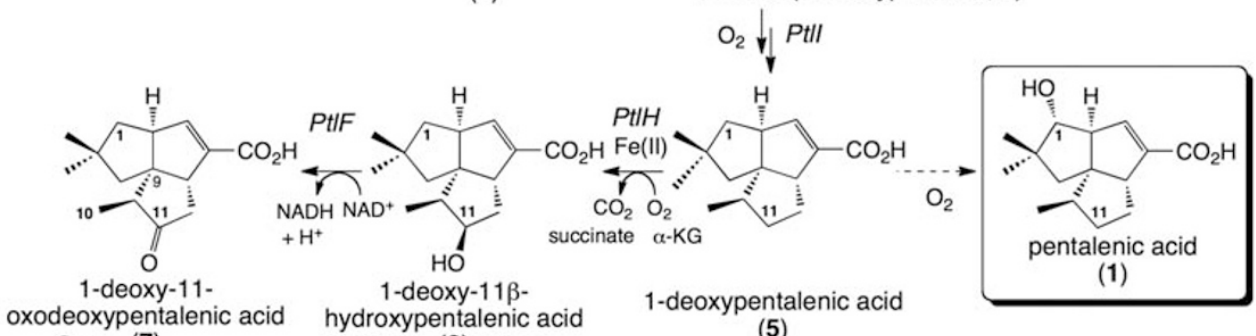

$\mathrm{O}_{2} \quad$ (7)

1-deoxy-11 $\beta$ -

(6) 1-deoxypentalenic acid

(5)

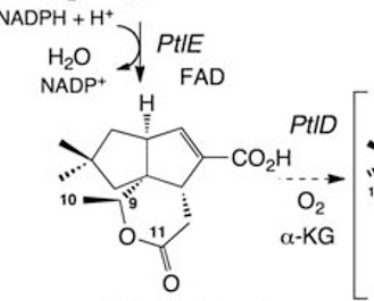

neopentalenolactone $\mathrm{D}$

(8)
NADPH+ $\mathrm{H}^{+}$<smiles>CC[C@]1(C)C[C@H]2C=C(C(=O)O)[C@@H]3CC(=O)OC[C@@]23[C@@H]1O</smiles>

neopentalenoketolactone

(9)

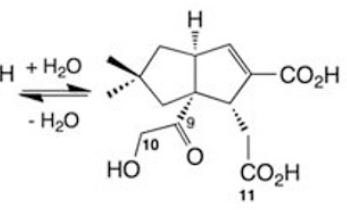

hydroxyketo seco acid

(10)

Figure 2 Proposed neopentalenoketolactone biosynthetic pathway in S. avermitilis. $\alpha$-KG indicates $\alpha$-ketoglutarate.

\section{5'-GCCCGCCGGGGAAAATCTCCGGGTGGGGGTTGGTCGTCACCCCGGG} TACCGAGCGAAC-3' (bold characters indicate the downstream region of $p t l H$ on the chromosome and underlined characters correspond to the stop codon of $p t l D)$. This primer pair was used for the amplification of the $\operatorname{lox} P$-aad( $\left.3^{\prime \prime}\right)-\operatorname{lox} P$ segment using pKU473 (pULwL:: aad $\left(3^{\prime \prime}\right)$ ) as template. ${ }^{19}$ The initial denaturation step $\left(95^{\circ} \mathrm{C}, 5 \mathrm{~min}\right)$ was followed by five cycles of amplification $\left(95^{\circ} \mathrm{C}\right.$, $\left.30 \mathrm{~s} ; 50{ }^{\circ} \mathrm{C}, 30 \mathrm{~s} ; 72^{\circ} \mathrm{C}, 60 \mathrm{~s}\right)$ followed by 25 cycles using an annealing temperature of $65^{\circ} \mathrm{C}$, and then a final incubation at $72{ }^{\circ} \mathrm{C}(10 \mathrm{~min})$. The desired in-frame deletion plasmid, pKU462::ptl-cluster $\Delta p t l H$, was constructed using the $\lambda$ recombination system and Cre/loxP-based site-specific excision, as previously described. ${ }^{19,21}$

Isolation of products from $S$. avermitilis and its deletion mutants The cultivation of in-frame deletion mutants was performed as previously described. ${ }^{14,19}$ After cultivation, an equal volume of methanol was added and mixed for $10 \mathrm{~min}$. The mycelia were sedimented by centrifugation, the supernatant was evaporated under reduced pressure to remove methanol. The aqueous extract was diluted with a half volume of water, adjusted to $\mathrm{pH} 2$ with $2 \mathrm{~N} \mathrm{HCl}$ and extracted twice with a half volume of EtOAc. The resulting organic extracts were combined, dried under $\mathrm{Na}_{2} \mathrm{SO}_{4}$ and evaporated to dryness. The brownish oily residue was dissolved in $0.1 \mathrm{ml}$ of benzenemethanol (9:1) and the products were methylated by adding $0.02 \mathrm{ml}$ of trimethylsilyldiazomethane10\% in $n$-hexane) for $15 \mathrm{~min}$ at ambient temperature. After removal of solvent by evaporation, the methylated products were re-dissolved in a small amount of methanol and a portion of the extract was subjected to gas chromatography-MS (GC-MS) analysis (Shimadzu GC-17A, $70 \mathrm{eV}$, electron impact, positive ion mode; $30 \mathrm{~m} \times 0.25 \mathrm{~mm} \phi$ Neutra Bond-5 capillary column (5\% phenylmethylsilicon) using a temperature program of $50-280^{\circ} \mathrm{C}$ and temperature gradient of $20^{\circ} \mathrm{C} \mathrm{min}^{-1}$, inlet temperature $280^{\circ} \mathrm{C}$; run time $20 \mathrm{~min}$ ).

Construction of the sav7469 expression cassette

As the sav7470 gene (8897117-8894401 nucleotide in the S. avermitilis genome) encoding a ferredoxin $(\mathrm{FdxH})$ is located 14-basepairs downstream of the $\operatorname{sav} 7469$ gene, the two genes appear to belong to a single transcriptional unit. An operon containing both sav7469 and sav7470 was amplified by PCR from template DNA from cosmid CL_228_E11 using the primer pair, forward: 5'-GCCCATATGACAGAGCCCGGTACGTCCGT-3' (underlined characters indicate the NdeI site and bold characters correspond to the start codon of sav7469) and reverse: 5'-GCACTAGTTCAGCTTGCCGACCGCGGGAC-3' (underlined characters indicate the SpeI site and bold characters correspond to the stop codon of sav7470). The amplified DNA segment was digested with $\mathrm{NdeI}$ and SpeI and the digested segment was ligated with the NdeI/XbaI fragment of pKU460::xylAp-sav3129-sav5675. ${ }^{24}$ The resultant recombinant plasmid pKU460::xylAp-sav7469-sav7470-sav3129-sav5675 was digested with $\mathrm{NdeI} / \mathrm{HindIII}$ and the resultant NdeI/HindIII segment sav7469-sav7470sav3129-sav5675 was ligated with the large segment of NdeI/HindIII pKU460::rpsIp. ${ }^{21}$ The plasmid isolated from the resulting transformants was digested with NheI/HindIII and the NheI/HindIII segment, rpsJp-sav7469sav7470-sav3129-sav5675, was ligated with the large segment of NheI/HindIII pKU 490:: $h p h$ and the ligation product was used to transform E. coli DH5 $\alpha$. The desired plasmid, pKU490::rpsJp-sav7469-sav7470-sav3129-sav5675, obtained by selection for hygromycin B resistance, was transferred into S. avermitilis SUKA13 carrying pKU462::ptl-cluster $\Delta p t l H$ by conjugation, as previously described. ${ }^{25}$

\section{Heterologous expression and purification of CYP105D7 (SAV_7469)}

The sav7469 gene ( $8895895-8897103 \mathrm{nt}$ in S. avermitilis) was amplified by PCR with template DNA from cosmid CL_228_E11 using the primer pair, forward: 5'-CGGAATTCCATATGACAGAGCCCGGTACGTCCGTGTC-3' (underlined characters indicate the NdeI site) and reverse: 5'-GGACTAGTTCAGTGGT GGTGGTGCCAGGTCACGGGGAGTTCCAGCATC-3' (underlined characters indicate the SpeI site and bold characters represent $\mathrm{His}_{4}$ tag). The PCR program employed was as follows: initial denaturation step $\left(96^{\circ} \mathrm{C}, 2 \mathrm{~min}\right)$, followed by 30 cycles of amplification $\left(96^{\circ} \mathrm{C}, 30 \mathrm{~s} ; 65^{\circ} \mathrm{C}, 30 \mathrm{~s}\right.$; and $\left.72{ }^{\circ} \mathrm{C}, 60 \mathrm{~s}\right)$, and then a final incubation at $72{ }^{\circ} \mathrm{C}(5 \mathrm{~min})$. The resultant amplicon and $\mathrm{pET17b}$ vector were each then double-digested with NdeI and SpeI. The digested PCR product was ligated 
with the large NdeI/SpeI fragment of pET17b by T4 DNA ligase. The ligation mixture was used to transform competent cells of E. coli JM109. The resultant plasmid, pET17b::sav7469, isolated from cultures grown on LA medium containing $50 \mu \mathrm{g} \mathrm{ml}^{-1}$ of ampicillin, was used to transform E. coli BL21 CodonPlus (DE3). Expression of the $\operatorname{sav} 7469$ gene and purification of $\mathrm{C}$-terminal $\mathrm{His}_{4}$-tagged CYP105D7 was performed by procedures similar to those described previously. ${ }^{26}$ The purified CYP105D7 $\left(10 \mathrm{mg} \mathrm{ml}^{-1}\right)$ was flash-frozen in small aliquots in liquid $\mathrm{N}_{2}$ and stored at $-80^{\circ} \mathrm{C}$. The measurement of UV-visible absorption spectrum under oxidized and reduced conditions, and CO-difference spectrum was performed by procedures described previously. ${ }^{27}$

\section{Preparation of 1-deoxypentalenic acid (5)}

The culture conditions for the production of 1-deoxypentalenic acid by $S$. avermitilis SUKA13 carrying pKU462::ptl-cluster $\Delta p t l H$ were described previously. ${ }^{19}$ Around 21 of the culture supernatant was acidified to $\mathrm{pH} 2.5$ with $2 \mathrm{~N} \mathrm{HCl}$ and the organic products were extracted twice with 11 of EtOAc. The organic extracts were combined, dried under $\mathrm{Na}_{2} \mathrm{SO}_{4}$ and evaporated to dryness. The brownish oily material was dissolved in $2 \mathrm{ml}$ of benzene-methanol (9:1) and treated with $0.1 \mathrm{ml}$ of trimethylsilyldiazomethane for $15 \mathrm{~min}$ at room temperature. After removal of solvent, methylated products were subjected to silica gel column chromatography using $\mathrm{CHCl}_{3}$ as eluent with collection of the (5) methyl ester-rich fraction. Pure (5)-methyl ester was obtained by preparative HPLC developing with $90 \%$ acetonitrile in water. The resulting (5)-methyl ester $(27 \mathrm{mg})$ was hydrolyzed with $5 \mathrm{~mm} \mathrm{~K} \mathrm{~K}_{2} \mathrm{CO}_{3}$ in $30 \%$ (v/v) aqueous methanol (reflux, $24 \mathrm{~h}$ ). After removal of the methanol under reduced pressure, the resultant aqueous solution was adjusted to $\mathrm{pH} 2.5$ with $2 \mathrm{~N} \mathrm{HCl}$ and extracted with equal volume of EtOAc to give $17 \mathrm{mg}$ of 1-deoxypentalenic acid (5) as a colorless oil. The structure of $\mathbf{5}$ was confirmed by the ${ }^{1} \mathrm{H}$ and ${ }^{13} \mathrm{C}$ NMR spectra.

\section{In vitro conversion of 1-deoxypentalenic acid (5) to pentalenic acid} (1) by CYP105D7

The catalytic reaction of CYP105D7 was performed using the following reaction mixture: $0.4 \mathrm{~mm}$ 1-deoxypentalenic acid (5) in DMSO, $4.4 \mu \mathrm{M}$ spinach $\mathrm{Fdx}, 0.05 \mathrm{U}$ of spinach Fdx-NADP ${ }^{+}$reductase, $0.5 \mathrm{~mm} \mathrm{NADP}^{+}, 0.5 \mathrm{~mm}$ glucose6-phosphate, $0.5 \mathrm{U}$ of glucose-6-phosphate dehydrogenase, $50 \mathrm{~mm}$ Tris- $\mathrm{HCl}$ $(\mathrm{pH} 8.0), 10 \%(\mathrm{v} / \mathrm{v})$ of glycerol and $1.1 \mu \mathrm{m}$ purified C-terminal $\mathrm{His}_{4}$-tagged CYP105D7, in a total volume of $200 \mu$ l. The reaction mixture without CYP105D7 was pre-incubated for $15 \mathrm{~s}$ at $25^{\circ} \mathrm{C}$ before addition of CYP105D7 and incubation for $2 \mathrm{~h}$ at $30^{\circ} \mathrm{C}$ with shaking. The enzymatic reaction was terminated by adding $20 \mu \mathrm{l}$ of $1 \mathrm{~N} \mathrm{HCl}$ and the product was extracted using $2 \times 100 \mu \mathrm{l}$ of EtOAc. The combined organic extracts were dried over $\mathrm{Na}_{2} \mathrm{SO}_{4}$ and evaporated to dryness. The residue was dissolved in $100 \mu$ of benzenemethanol (9:1) and $20 \mu \mathrm{l}$ of trimethylsilyldiazomethane was added. After $15 \mathrm{~min}$ at room temperature, the reaction mixture was evaporated to dryness and the residue was dissolved in $20 \mu \mathrm{l}$ methanol. A $5-\mu \mathrm{l}$ portion of the methylated product was analyzed by GC-MS. For kinetic measurements, assays were performed under the above conditions over a range of substrate concentrations. The steady-state kinetic parameters, $K_{\mathrm{m}}, V_{\max }$ and $k_{\text {cat }}$, were calculated by fitting the observed GC-MS TIC values to the Michaelis-Menten equation by non-linear, least-squares regression. Reported standard deviations correspond to the statistical errors limits of the data fit. The dissociation constant $K_{\mathrm{D}}$ of CYP105D7 and 1-deoxypentalenic acid (5) was obtained as previously described..$^{15}$ 1-Deoxypentalenic acid (5) dissolved in DMSO was added at increasing concentrations to a stock solution of $1.1 \mu \mathrm{M}$ of CYP105D7 in $50 \mathrm{~mm}$ Tris- $\mathrm{HCl}(\mathrm{pH} 8.0)$ and $10 \%(\mathrm{v} / \mathrm{v})$ glycerol. The concentration of DMSO was adjusted to $0.1 \%(\mathrm{v} / \mathrm{v})$. UV difference spectra were recorded from 0 to $100 \mu \mathrm{M}$ of 1-deoxypentalenic acid (5). The dissociation constant $K_{\mathrm{D}}$ was calculated from the saturation curve of the measured delta value $(\triangle \mathrm{AU}=\mathrm{A} 364 \mathrm{~nm}-\mathrm{A} 416 \mathrm{~nm})$ versus substrate concentration. All kinetics and dissociation experiments were run in triplicate.

\section{RESULTS}

Production of pentalenic acid (1) in mutants of $S$. avermitilis S. avermitilis harbors a biosynthetic gene cluster for a newly discovered branch of the classical pentalenolactone family tree, with the microorganism producing a new type of pentalenolactone derivative, which

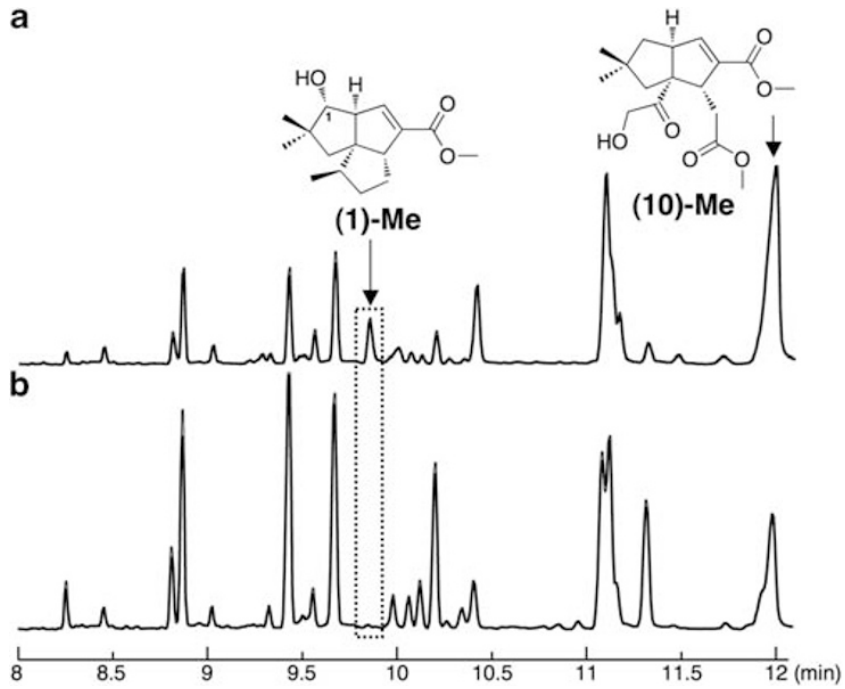

Figure 3 GC-MS analysis of EtOAc extracts treated with trimethylsilyldiazomethane of $S$. avermitilis SUKA5 (a) and SUKA7 (SUKA5 $\Delta$ sav7469:: $\operatorname{aadA}(\mathbf{b})$. The two strains were cultured at $28^{\circ} \mathrm{C}$ with shaking at 200 r.p.m. for $96 \mathrm{~h}$.

we have termed neopentalenolactone. ${ }^{19}$ One of the large-deletion mutants of $S$. avermitilis, SUKA5, produced pentalenic acid (1) and neopentalenoketolactone (9), which underwent facile conversion to the hydroxyketo seco acid form (10) (Figure 3a). We had independently constructed sav7469-deletion mutants for the bioconversion experiments. The mutant SUKA7, which harbors a deletion of the gene encoding CYP105D7 (SAV_7469), still produced neopentalenoketolactone (9) and its hydrolyzed derivative (10) but did not produce pentalenic acid (1) (Figure 3b). This result indicates that CYP105D7 is most likely involved in the formation of pentalenic acid (1) in S. avermitilis. To clarify the role of CYP105D7 in the hydroxylation of 1-deoxypentalenic acid (5) at C-1, a biosynthetically blocked mutant with a disrupted $p t l H$ (sav2991) gene, ${ }^{16}$ encoding a non-heme iron, $\alpha$-ketoglutarate-dependent hydroxylase that catalyzes the hydroxylation of 1-deoxypentalenic acid (5) at C-11 to generate 1-deoxy$11 \beta$-hydroxypentalenic acid (6), was constructed by in-frame deletion using the $\lambda$ recombination and Cre/loxP excision systems. ${ }^{19}$ As the C-11 hydroxylation was not functional in SUKA11 carrying pKU462:: ptl-cluster $\Delta p t l H$, this deletion mutant produced 1-deoxypentalenic acid (5) accompanied by relatively large amounts of pentalenic acid (1) (Figure 4a). Furthermore, when the sav7469-deletion was introduced into the SUKA11 carrying pKU462::ptl-cluster $\Delta p t l H$ mutant the resultant double-deletion mutant, SUKA13 carrying pKU462:: ptl-cluster $\Delta p t l H$, accumulated only 1-deoxypentalenic acid (5) (Figure 4b). Pentalenic acid (1) production could be restored by introduction of an extra copy of sav7469, which was co-expressed along with two genes encoding the $S$. avermitilis electron-transport partners, Fdx (SAV_3129; FdxD) and Fdx reductase (SAV_5675; FprD) into the double-deletion mutant (Figure 4c), because the production of oxidative product(s) of epi-isozizaene derivatives were enhanced by co-expression of these two genes with sav3032-sav3031. ${ }^{24}$ These results indicate that pentalenic acid (1) is generated in S. avermitilis by hydroxylation of 1-deoxypentalenic acid (5) at C-1 catalyzed by CYP105D7.

\section{Characterictics of CYP105D7}

From a BLAST homology search using the NCBI nr database, S. avermitilis CYP105D7 was found to be similar at the amino-acid 
a

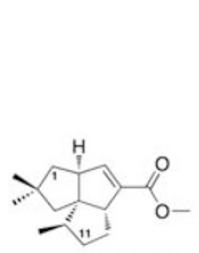

(5)-Me

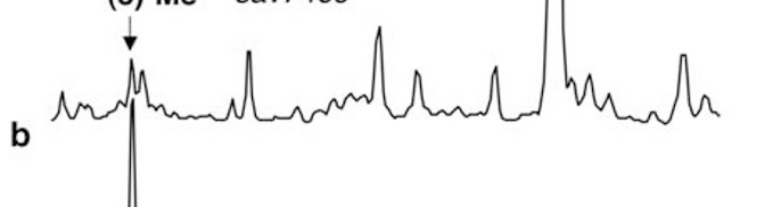

$\operatorname{sav7469}$

c

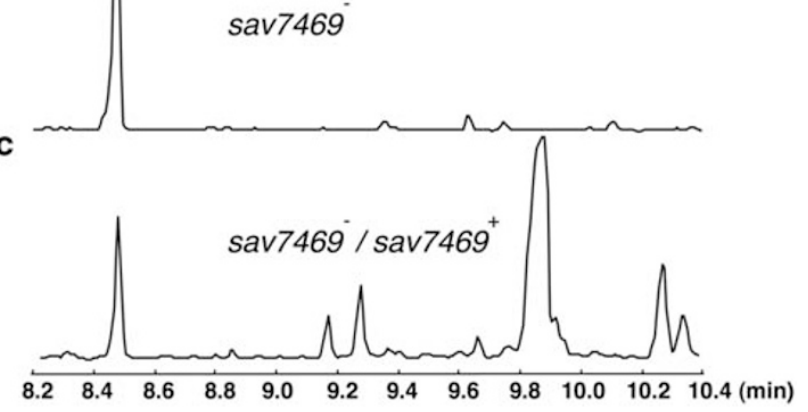

Figure 4 GC-MS analysis of EtOAc extracts treated with trimethylsilyldiazomethane of SUKA11 carrying pKU462:: ptl-cluster $\Delta p t / H$ (a), SUKA13 (SUKA11 $\Delta$ sav7469::aadA) carrying pKU462::ptl-clusterAptIH (b) and SUKA13 carrying pKU462::ptl-cluster $\Delta$ pt/H and pKU493::rpsJp-sav7469sav7470-sav3129-sav5675 (c).

(aa) sequence level to ZP_05504847 of Streptomyces sp. C (395 aa, 77\% identity and $86 \%$ positive matches), BAG55292 of Streptomyces sp. A-1544 (409 aa, 75\% identity and 87\% positive matches), ZP_05536090 of S. viridochromogenes DSM 40736 (398 aa, 75\% identity and 86\% positive matches), ZP_05021349 of S. sviceus Americam Type Culture Collection 29083 ( 413 aa, $75 \%$ identity and $85 \%$ positive matches), ZP_04683686 of S. ghanaensis Americam Type Culture Collection 14672 (401 aa, 73\% identity and 84\% positive matches), ZP_05528097 (CYP105D4) of S. lividans TK24 (406aa, 74\% identity and 85\% positive matches), NP_625076 (CYP105D5) of S. coelicolor A3(2) (412 aa, $74 \%$ identity and $85 \%$ positive matches), P26911 (CYP105D1) of S. griseus (412 aa, 71\% identity and $80 \%$ positive matches) and BAG50412 of S. griseolus (395 aa, 69\% identity and 80\% positive matches).

To confirm the predicted function of CYP105D7, the recombinant protein was expressed in the T7-RNA polymerase-based expression host E. coli BL21 CodonPlus (DE3). As induction with IPTG (0.1$0.5 \mathrm{~mm}$ ) was not effective for the expression of sav7469, the cultivation for the E. coli transformant carrying pET17b::sav7469 was performed without IPTG induction. The soluble C-terminal His H-tagged $_{4}$ CYP105D7 was purified by sequential $\mathrm{Ni}^{2+}$-affinity chromatography, ion-exchange chromatography and gel filtration chromatography. The recovered deep red recombinant CYP105D7 (15 mg from 21 of culture) was judged to be more than $90 \%$ pure by SDS-PAGE.

The UV-visible absorption spectrum of the oxidized-form of recombinant CYP105D7 showed a Soret peak plus $\beta$ - and $\alpha$-bands at 418,534 and $568 \mathrm{~nm}$, respectively, suggesting a typical low-spin state spectrum (Figure 5a). The relatively sharp peak at $447 \mathrm{~nm}$ in the CO-difference spectrum of recombinant CYP105D7 confirmed that
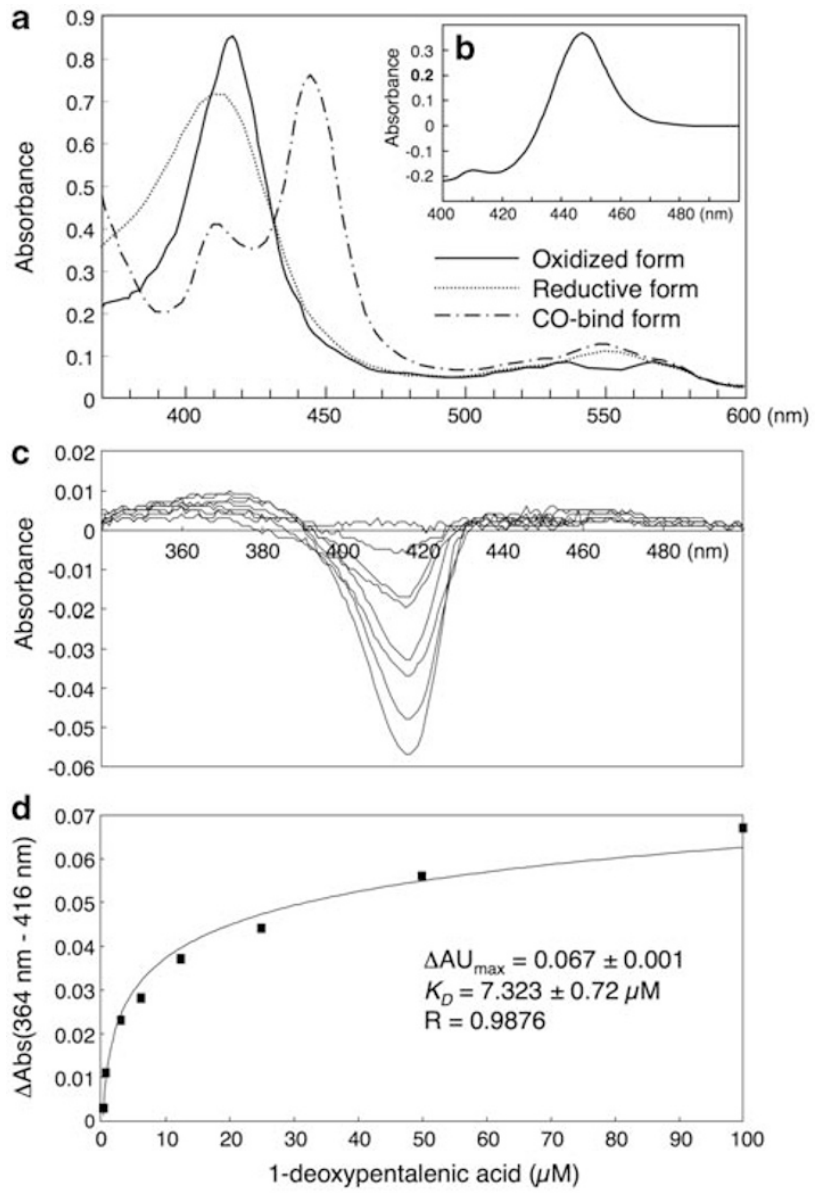

Figure 5 UV spectra of oxidized, reduced and CO-bound forms of C-terminal $\mathrm{His}_{4}$-tagged CYP105D7 (a) and its CO-difference spectrum (b). UV difference binding spectra of CYP105D7 and 1-deoxypentalenic acid (5) binding (c), saturation curve for CYP105D7 and 1-deoxypentalenic acid (5) (d).

the protein was correctly folded (Figure $5 b$ ). The difference spectra showed a typical type I spectral shift and positive peaks were observed in the difference binding spectra at 364 and $416 \mathrm{~nm}$ recorded at increasing concentrations of 5 (Figure $5 \mathrm{c}$ ). The plot of the measured delta value $(\triangle \mathrm{AU}=\mathrm{A} 364 \mathrm{~nm}-\mathrm{A} 416 \mathrm{~nm})$ versus substrate concentration is shown in Figure $5 \mathrm{~d}$. The $\Delta \mathrm{AU}_{\max }$ and a $K_{\mathrm{D}}$ values were calculated as $0.067 \pm 0.001$ and $7.3 \pm 0.7 \mu \mathrm{M}$, respectively.

In vitro hydroxylation of 1-deoxypentalenic acid (5) to pentalenic acid (1) catalyzed by recombinant CYP105D7

The reaction mixture was incubated in the presence of $2 \mu \mathrm{M}$ recombinant CYP105D7 for $2 \mathrm{~h}$ at $30^{\circ} \mathrm{C}$ with shaking. The substrate 1deoxypentalenic acid (5) was almost completely consumed, based on the disappearance of the GC-MS peak (ret. time $8.458 \mathrm{~min}$ ) corresponding to 5-methyl ester, whereas the formation of the product pentalenic acid (1) was evident from the appearance of a methyl ester peak (1-methyl ester), retention time $9.883 \mathrm{~min}$. In a parallel control incubation carried out in the absence of CYP105D7, there was no observable hydroxylation of 5 to $\mathbf{1}$. (Figure 6a). The EI-MS fragmentation pattern and the retention time of the peak corresponding to pentalenic acid (1) methyl ester were identical to those of an authentic sample (Figure 6b).

The $\mathrm{pH}$-dependence of the CYP105D7-catalyzed reaction was measured at $\mathrm{pH} 6.0,6.5,7.0,7.5$ and 8.0. The maximum rate of 


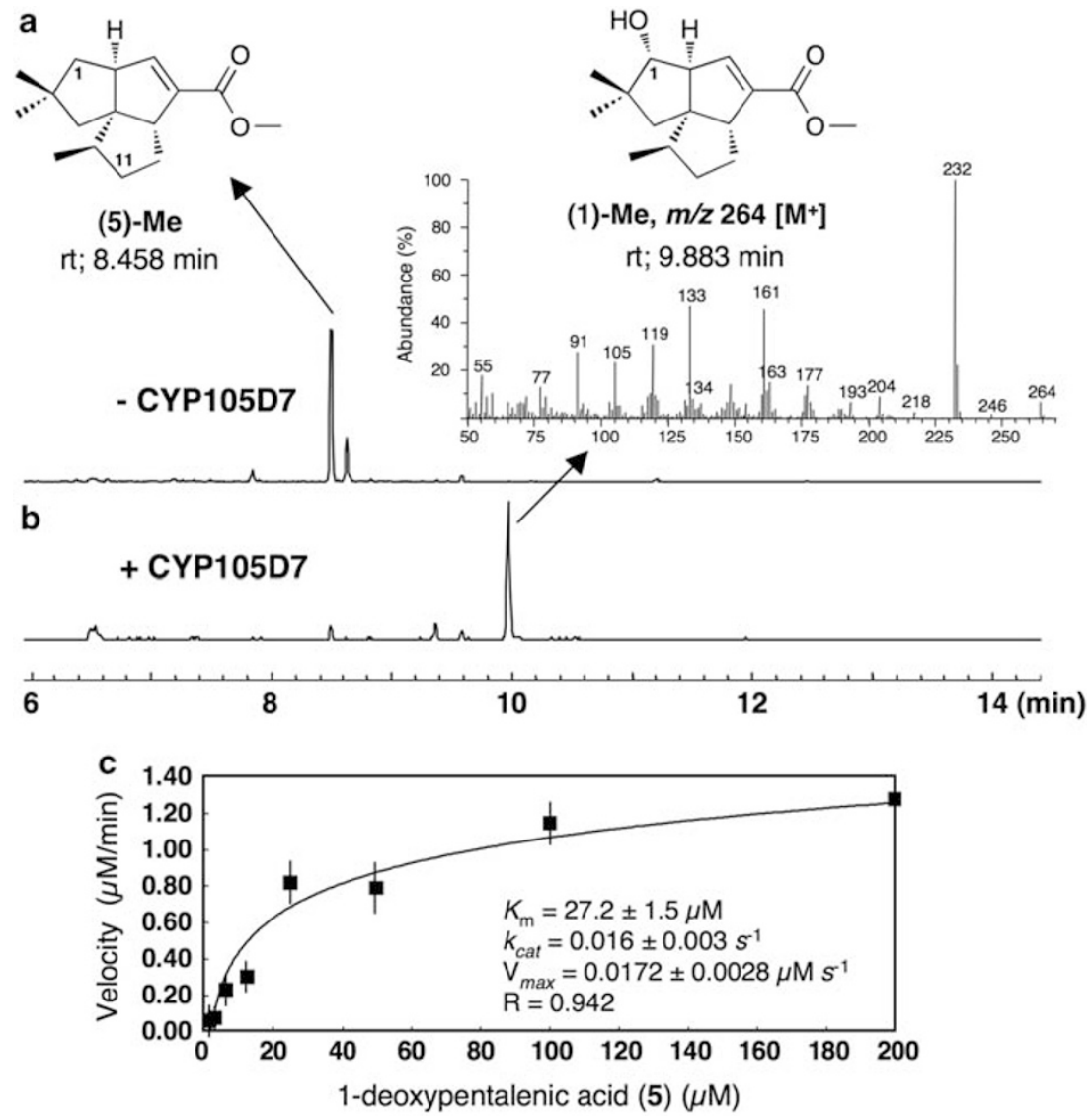

Figure 6 GC-MS analysis of EtOAc extracts treated with trimethylsilyldiazomethane from incubation of 1-deoxypentalenic acid (5) with (b) or without (a) CYP105D7 protein in the presence of a NADPH-generating system, electron-transport partners (spinach Fdx and Fdx reductase) and Michaelis-Menten plot of initial velocity of formation of pentalenic acid (1) (c).

formation of pentalenic acid (1) was observed at $\mathrm{pH} 8.0$ (data not shown). The steady-state kinetic parameters of the CYP105D7catalyzed hydroxylation reaction were $K_{\mathrm{m}}(5)$ of $27.2 \pm 2.5 \mu \mathrm{M}, V_{\max }$ $0.0172 \pm 0.003 \mu \mathrm{M} \mathrm{s}^{-1}$ and $k_{\text {cat }} 0.116 \pm 0.003 \mathrm{~s}^{-1}$ (Figure $6 \mathrm{c}$ ). To examine the substrate specificity of CYP105D7, several intermediates of pentalenolactone biosynthesis were incubated with CYP105D7. The recombinant CYP105D7 protein could not catalyze the C-1 hydroxylation of pentalenene (2), 1-deoxy-11 $\beta$-hydroxypentalenic acid (6), 1-deoxy-11-oxopentalenic acid (7) or the corresponding methyl esters of 6, 7 and 5, respectively. CYP105D7 is, therefore, specific for the C-1 hydroxylation of 1-deoxypentalenic acid (1).

\section{DISCUSSION}

The wild-type strain of $S$. avermitilis and its large-deletion derivative SUKA5, as well as S. lividans 1326 carrying the entire ptl cluster of S. avermitilis, each produce pentalenic acid (1), a known shunt metabolite in the biosynthesis of the pentalenolactone family of antibiotics. ${ }^{9}$ In principle, pentalenic acid (1) might be formed by the hydroxylation of 1-deoxypentalenic acid (5) at C-1 or the oxidation of pentalenene (2) at both C-1 and C-13. Pentalenic acid (1) is normally isolated as a common co-metabolite of pentalenolactone and its derivatives, including the biosynthetic intermediate, 1-deoxypentalenic acid (5). ${ }^{28}$ Although at least four genes in the S. avermitilis ptl cluster have been shown to encode oxygenases implicated in the biosynthesis of neopentalenoketolactone biosynthesis, the gene responsible for the formation of pentalenic acid (1) by hydroxylation of 1-deoxypentalenic acid (5) is not present in the ptl cluster. Therefore, we sought to determine which of the 33 CYP genes that have been identified in the genome of $S$. avermitilis would be responsible for the generation of pentalenic acid (1). The altered product profile in the SUKA7 large-deletion mutant that lacks the sav7469 gene encoding CYP105D7 indicates that production of pentalenic acid (1) depends on the presence of sav7469 gene. This relationship was further examined and supported using biosynthetically blocked mutants of the large-deletion mutants SUKA5 and SUKA7.

It is well-known that CYP105 family monooxygenases have a remarkable capacity for broad-based xenobiotic metabolism, utilizing compounds of diverse structure and chemistry. ${ }^{20,29}$ In the $p t l$ cluster for the biosynthesis of neopentalenoketolactone, the only CYP gene involved in the formation of an intermediate for neopentalenolactones, is ptlI (sav2999) the gene product (CYP183A1) of which has been shown to oxidize the C-13 methyl of pentalenene (2) to give 1deoxypentalenic acid (5). ${ }^{15}$ As no further metabolites of pentalenic acid (1) have been isolated from S. avermitilis, pentalenic acid (1) was thought to be formed by direct hydroxylation of 1-deoxypentalenic acid (5) catalyzed by CYP105D7. Notably, this C-1 oxidation involves removal of a different diastereotopic proton from that which is lost in the final oxidative rearrangement that results in the formation of pentalenolactone. ${ }^{9}$

The in vitro enzymatic reaction unambiguously established that CYP105D7 is responsible for the conversion of 1-deoxypentalenic acid (5) to pentalenic acid (1). Although CYP105D7 has been shown to have a relatively broad substrate range, being able to catalyze the hydroxylation of milbemycin A5, compactin, diclofenac, lauric acid 
and narigenin (unpublished data), no pentalenolactone-related compounds, other than 1-deoxypentalenic acid (5), could be hydroxylated by CYP105D7. As pentalenene (2) was not oxidized by CYP105D7, the formation of the shunt metabolite pentalenic acid (1) in S. avermitilis is conclusively shown to involve sequence conversion of farnesyl diphosphate to pentalenene (2) by pentalenene synthase, PtlI-catalyzed oxidation of 2 to 1-deoxypentalenic acid (5), and finally CYP105D7-catalyzed hydroxylation of $\mathbf{5}$ to pentalenic acid (1). We have previously demonstrated that exoconjugants of S. lividans 1326 carrying the $S$. avermitilis ptl cluster produced only pentalenic acid (1). ${ }^{14}$ As S. lividans also possesses a gene encoding a member of the CYP105D subfamily of monooxygenases, CYP105D $4,{ }^{20}$ the S. lividans exoconjugant carrying the ptl cluster most likely generated 1-deoxypentalenic acid (5), which presumably served as the substrate for hydroxylation by CYP105D4 to pentalenic acid (1). Genes encoding CYP105D subfamily monooxygenases have been found in all genome-sequenced Streptomyces, including draft genome sequenced microorganisms. It is, therefore, likely that other pentalenolactoneproducing Streptomyces strains might possess the similar genes encoding members of the CYP105D subfamily of monooxygenases. Accumulation of the shunt product pentalenic acid (1) in pentalenolactone-producing microorganisms would, therefore, result from the abortive hydroxylation of the normal 1-deoxypentalenic acid (5) intermediate by CYP105D subfamily monooxygenase in the producing microorganism. Streptomyces exfoliates UC5319 also accumulates pentalenolactone $\mathrm{H}$ as a minor component, derived by $\mathrm{C}-1$ hydroxylation of pentalenolactone $\mathrm{F}^{9}$ It is likely that a specific CYP105D subfamily monooxygenase in this microorganism might be able to catalyze the requisite oxidation of pentalenolactone $\mathrm{F}$.

\section{ACKNOWLEDGEMENTS}

This work was supported by a Grant-in-Aid for Scientific Research on Innovative Areas from the Ministry of Education, Culture, Sports, Science and Technology, Japan, a Grant-in-Aid from the Japan Society for the Promotion of Science (JSPS) 20310122 and a research grant of the Institute for Fermentation, Osaka, Japan (HI) and by NIH Grant GM30301 (DEC).

1 Koe, B. K., Sobin, B. A. \& Celmer, W. D. PA 132, a new antibiotic. I. Isolation and chemical properties. Antibiot. Annu. 672-675 (1957).

2 Martin, D. G., Slomp, G., Mizsak, S., Duchamp, D. J. \& Chidester, C. G. The structure and absolute configuration of pentalenolactone (PA 132). Tetrahedron Lett. 11, 4901-4904 (1970).

3 Keller-Schierlein, W., Lemke, J., Nyfeler, R. \& Zähner, H. Stoffwechelprodukte von Mikroorganismen. 105. Arenaemycin E, D, und C. Arch. Mikrobiol. 84, 301-316 (1972).

4 Takahashi, S., Takeuchi, M., Arai, M., Seto, H. \& Otake, N. Studies on the biosynthesis of pentalenolactone. V. Isolation of deoxypentalenylglucuron. J. Antibiot. 36, 226-228 (1983).

5 English, A. R., McBride, T. J. \& Lynch, J. E. PA 132, a new antibiotic. II. In vitro and in vivo studies. Antibiot. Annu. 682-687 (1957).
6 Nakagawa, A. et al. Antiviral activities of pentalenolactones. J. Antibiot. 38, 1114-1115 (1985).

7 Ikeda, M., Fukuda, A., Takagi, M., Morita, M. \& Shimada, Y. Inhibitory effect of pentalenolactone on vascular smooth muscle cell proliferation. Eur. J. Pharm. 411, 45-53 (2001).

8 Seto, H., Sasaki, T., Uzawa, J., Takeuchi, S. \& Yonehara, H. Studies on the biosynthesis of pentalenolactone. Part II. Isolation of pentalenic acid and pentalenolactone $\mathrm{H}$. Tetrahedron Lett. 19, 4411-4412 (1978).

9 Cane, D. E. et al. The biosynthesis of pentalenene and pentalenolactone. J. Am. Chem. Soc. 112, 4513-4524 (1990).

$10 \mathrm{lkeda}, \mathrm{H}$. et al. Complete genome sequence and comparative analysis of the industrial microorganism Streptomyces avermitilis. Nat. Biotechnol. 21, 526-531 (2003).

11 Nett, M., Ikeda, H. \& Moore, B. S. Genomic basis for natural product biosynthetic diversity in the actinomycetes. Nat. Prod. Rep. 26, 1362-1384 (2009).

12 Cane, D. E., He, X., Kobayashi, S., Omura, S. \& Ikeda, H. Geosmin biosynthesis in Streptomyces avermitilis. Molecular cloning, expression and mechanistic study of the Germacradienol/Geosmin synthase. J. Antibiot. 59, 471-479 (2006).

13 Wayne, K. W. et al. Genome mining in Streptomyces avermitilis: cloning and characterization of SAV_76, the synthase for a new sesquiterpene. Avermitilol. J. Am. Chem. Soc. 132, 8850-8851 (2010).

14 Tetzlaff, C. N. et al. A gene cluster for biosynthesis of the sesquiterpenoid antibiotic pentalenolactone in Streptomyces avermitilis. Biochemistry 45, 6179-6186 (2006).

15 Quaderer, R., Omura, S., Ikeda, H. \& Cane, D. E. Pentalenolactone biosynthesis. Molecular cloning and assignment of biochemical function to PtII, a cytochrome P450 of Streptomyces avermitilis. J. Am. Chem. Soc. 128, 13036-13037 (2006).

16 You, Z., Omura, S., Ikeda, H. \& Cane, D. E. Pentalenolactone biosynthesis. Molecular cloning and assignment of biochemical function to $\mathrm{PtIH}$, a non-heme iron dioxygenase of Streptomyces avermitilis. J. Am. Chem. Soc. 128, 6566-6567 (2006).

17 You, Z., Omura, S., Ikeda, H., Cane, D. E. \& Jogl, G. Crystal structure of the non-heme iron dioxygenase PtIH in pentalenolactone biosynthesis. J. Biol. Chem. 282, 36552-36560 (2007)

18 You, Z., Omura, S., Ikeda, H. \& Cane, D. E. Pentalenolactone biosynthesis: molecular cloning and assignment of biochemical function to PtIF, a short-chain dehydrogenase from Streptomyces avermitilis, and identification of a new biosynthetic intermediate. Arch. Biochem. Biophys. 459, 233-240 (2007).

19 Jiang, J. et al. Genome mining in Streptomyces avermitilis: a biochemical BaeyerVilliger reaction and discovery of a new branch of the Pentalenolactone family tree. Biochemistry 48, 6431-6440 (2009).

20 Lamb, D. C. et al. Cytochrome p450 complement (CYPome) of the avermectin-producer Streptomyces avermitilis and comparison to that of Streptomyces coelicolor $\mathrm{A} 3(2)$. Biochem. Biophys. Res. Commun. 307, 610-619 (2003).

21 Komatsu, M., Uchiyama, T., Omura, S., Cane, D. E. \& Ikeda, H. Genome-minimized Streptomyces host for the heterologous expression of secondary metabolism. Proc. Natl. Acad. Sci. USA 107, 2646-2651 (2010).

22 Ikeda, H., Kotaki, H. \& Omura, S. Genetic studies of avermectin biosynthesis in Streptomyces avermitilis. J. Bacteriol. 169, 5615-5621 (1987).

23 Ikeda, H., Takada, Y., Pang, C. H., Tanaka, H. \& Omura, S. Transposon mutagenesis by Tn4560 and applications with avermectin-producing Streptomyces avermitilis. J. Bacteriol. 175, 2077-2082 (1993).

24 Takamatsu, S. et al. Characterization of a silent sesquiterpenoid biosynthetic pathway in Streptomyces avermitilis controlling epi-isozizaene and albaflavenone biosynthesis and isolation of a new oxidized epi-isozizaene metabolite. Microb. Biotechnol. (doi:10.1111/j.1751-7915.2010.00209.x).

25 Komatsu, M., Tsuda, M., Omura, S., Oikawa, H. \& Ikeda, H. Identification and functional analysis of genes controlling biosynthesis of 2-methylisoborneol. Proc. Natl. Acad. Sci. USA 105, 7422-7427 (2008).

26 Xu, L. H., Fushinobu, S., Ikeda, H., Wakagi, T. \& Shoun, H. Crystal structures of cytochrome P450 105P1 from Streptomyces avermitilis: conformational flexibility and histidine ligation state. J. Bacteriol. 191, 1211-1219 (2009).

27 Omura, T. \& Sato, R. The carbon monoxide-binding pigment of liver microsomes. J. Biol. Chem. 239, 2370-2378 (1964).

28 Cane, D. E., Sohng, J. K. \& Williard, P. G. Isolation and structure determination of pentalenolactones A, B, D, and F. J. Am. Chem. Soc. 57, 844-851 (1992).

29 Lamb, D. C. et al. The cytochrome P450 complement (CYPome) of Streptomyces coelicolor A3(2). J. Biol. Chem. 277, 24000-24005 (2002). 